EPJ Web of Conferences 81, 06007 (2014)

DOI: $10.1051 /$ epjconf/201481 06007

(C) Owned by the authors, published by EDP Sciences, 2014

\title{
Investigation of three nucleon force effects in deuteron-proton breakup reaction
}

W. Parol ${ }^{1, \text { a }}$, A. Kozela ${ }^{2}$, I. Ciepał ${ }^{1}$, B. Kłos ${ }^{3}$, K. Bodek$^{1}$, J. Golak ${ }^{1}$, N. Kalantar-Nayestanaki ${ }^{4}$, G. Khatri ${ }^{1}$, St. Kistryn ${ }^{1}$, P. Kulessa ${ }^{2}$, A. Magiera ${ }^{1}$, I. Mazumdar ${ }^{5}$, J. Messchendorp ${ }^{4}$, D. Rozpędzik ${ }^{1}$, R. Skibiński ${ }^{1}$, I. Skwira-Chalot ${ }^{6}$, E. Stephan ${ }^{3}$, H. Witała ${ }^{1}$, A. Wrońska ${ }^{1}$, and J. Zejma ${ }^{1}$

${ }^{1}$ Institute of Physics, Jagiellonian University, Kraków, Poland

${ }^{2}$ Institute of Nuclear Physics PAN, Kraków, Poland

${ }^{3}$ Institute of Physics, University of Silesia, Katowice, Poland

${ }^{4} \mathrm{KVI}$-CART, University of Groningen, Groningen, The Netherlands

${ }^{5}$ Tata Institute of Fundamental Research, Mumbai, India

${ }^{6}$ Faculty of Physics, University of Warsaw, Warsaw, Poland

\begin{abstract}
Experimental study of the deuteron-proton breakup process was perfomed in KVI Groningen. In this paper current status of the collected data analysis is presented, including preliminary results of the cross section for the sample kinematical configuration.
\end{abstract}

\section{Introduction}

Investigation of three-nucleon systems provides basis for the understanding of effective nucleonnucleon $(N N)$ potential and dynamics beyond such pairwise interactions. Quantitatively, this can be done by comparing observables calculated with the use of Faddeev equations with results of precise measurements. Modern realistic $N N$ interaction models describe well systems composed of two nucleons. Calculations based on these models could correctly predict observables for $3 N$ systems only if combined with additional component of the dynamics - the three nucleon force ( $3 N F)$ [1]. Threenucleon interaction can also be introduced within the coupled-channel (CC) framework by an explicit treatment of the $\Delta$-isobar excitation [2]. Alternatively, contribution of $N N$ and $3 N F$ to the potential energy of a $3 N$ system can be calculated within Chiral Perturbation Theory [3]. Here, the many-body interactions appear naturally at higher orders (non-vanishing $3 N F$ at next-to-next- to leading order). Modern calculations include also other ingredients of few nucleon dynamics such as Coulomb interactions [4] or relativistic effects [5]. Predicted effects in differential cross section appear in different parts of the phase space of the deuteron-proton breakup reaction with different magnitude. Existing experimental data (see e.g. [6], [7], [8]) demonstrate quite sizable $3 N F$ and Coulomb effects in, and confirm their importance for correct description of observables for the breakup reaction at medium energies. Such studies are continued at different energies in order to create the systematic database for testing models of the $3 N$ system dynamics.

a. e-mail: wiktor.parol@gmail.com 


\section{Experimental setup}

The experiment was performed at KVI Groningen with the use of the BINA detection system [7], dedicated to investigate few-body system dynamics in an almost $4 \pi$ geometry. BINA was installed on the beamline of AGOR with a beam energy of $160 \mathrm{MeV}$ impinging on hydrogen target. Charged products originating from elastic scattering and ${ }^{1} \mathrm{H}(\mathrm{d}, \mathrm{pp}) \mathrm{n}$ breakup reaction channels were detected simultaneously. The backward part of the system (BALL), made of $2 \times 149$ plastic scintillators working in a phoswich mode, covers a range of polar angles between $40^{\circ}$ and $165^{\circ}$ and a full range of azimuthal angles. The forward part (WALL) consists of the Multi-Wire Proportional Chamber (MWPC) and two layers of plastic scintillator hodoscopes: one $12 \mathrm{~cm}$ thick, for total energy reconstruction $(E)$ and one thin $(2 \mathrm{~mm})$, for energy loss information $(\Delta E)$. The WALL part accepts particles within the polar angles from $13^{\circ}$ to $40^{\circ}$. The MWPC provides resolution of $0.4^{\circ}$ in polar and $0.6^{\circ}-2.0^{\circ}$ in azimuthal angles, depending on polar angle.

\section{Data analysis}

The collected data were preliminary sorted by removing those measured in periods characterized by unstable beam current or affected by malfunctions of the system elements. Next, the energy calibration and particle identification were performed [9]. For a better control of systematic effects two methods of setting particle identification (PID) cuts were proposed. Both are based on $\Delta E-E$ technique applied to each virtual telescope (an overlap of a $\Delta E$ stripe with an $E$ slab) separately. In the first approach, bands corresponding to protons and deuterons in a given $\Delta E-E$ spectrum were defined manually (so-called graphical cuts were applied), whereas in the second case a semi-automatic linearization method [10] was used (see Fig. 1 and Fig. 2). For the discrimination of elastic scattering events from the breakup events, beside the particle identification, the coplanarity condition $\left(\left|\phi_{1}-\phi_{2}\right| \sim 180^{\circ}\right)$ and cut on an energy (selection of the peak of protons originating from the elastic scattering) were used.

The aim of the experiment is to obtain differential cross section for the breakup reaction for a large set of angular configurations on systematic grid defined by polar angles of the two protons
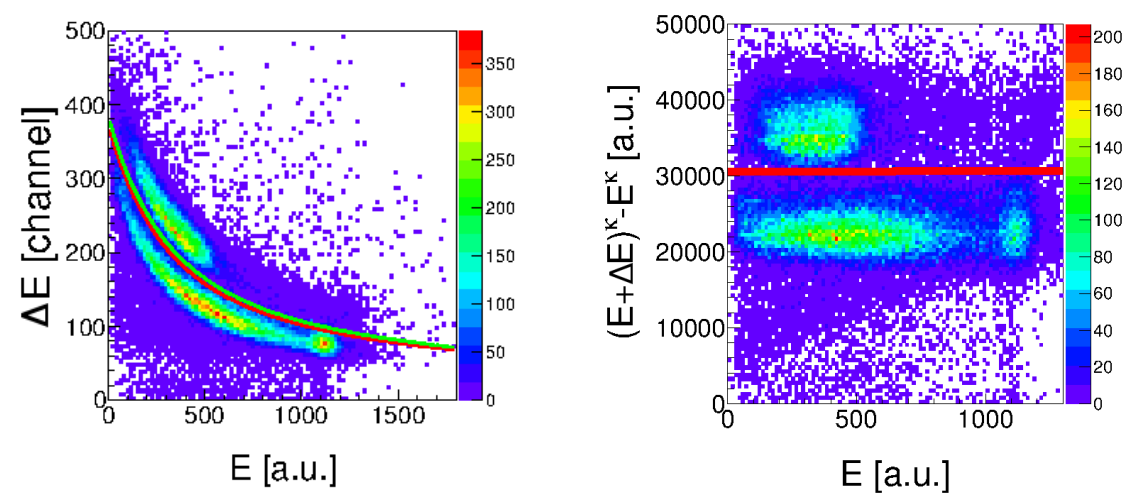

Figure 1. Data from deuteron-proton and deuteron-deuteron reactions registered in the sample $\Delta E-E$ telescope before (left panel) and after applying of the linearization function $(E+\Delta E)^{\kappa}-E^{\kappa}$ (right panel). 
MESON 2014 $-13^{\text {th }}$ International Workshop on Production, Properties and Interaction of Mesons
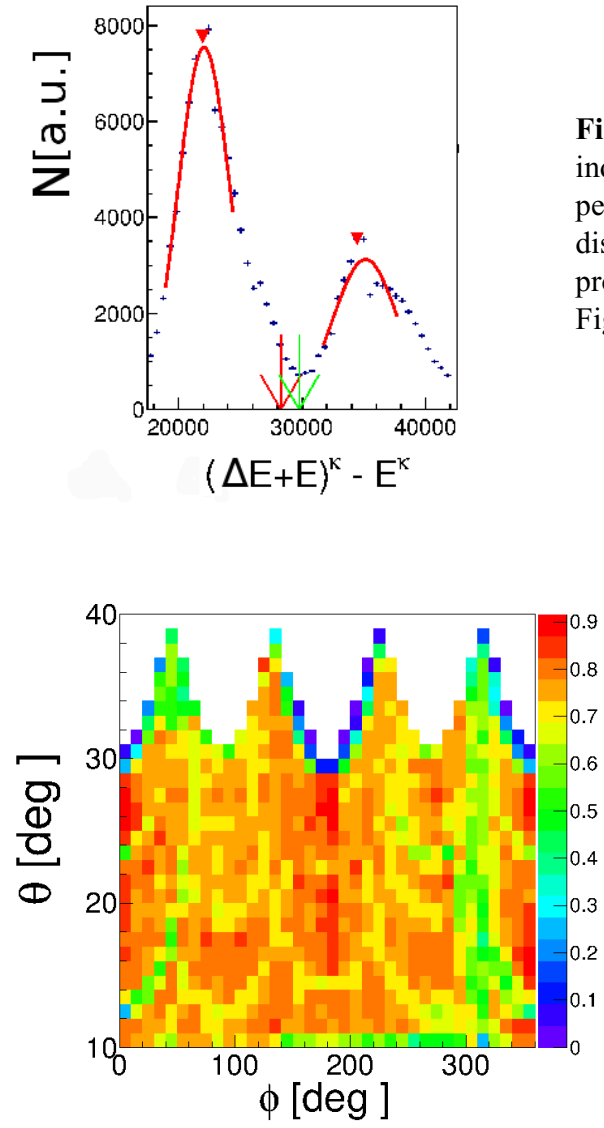

Figure 2. The $\kappa$ parameter value was calculated for each telescope individually to obtain best separation of proton and deuteron peaks, seen in $y$-axis projection, approximated with two gaussian distributions. Position of the peaks (red triangles) defines the protons and deuterons bounds in linearized $\Delta E-E$ spectrum (see Fig. 1), right panel
Figure 3. Efficiency map for protons detection as a product of Multi-Wire Proportional Chamber efficiency $\left(\epsilon_{M W P C}^{p}\right)$ and $\Delta E$ detector efficiency $\left(\epsilon_{\Delta E}^{p}\right)$.

$\left(\theta_{1}, \theta_{2}\right)$ and their relative azimuthal angle $(\Delta \phi)$. For each configuration the cross-section is obtained as a function of the arc-length $S$ along a theoretical kinematical curve corresponding to the point-like, central geometry. To obtain this function, the data accumulated in the 2-dimensional kinematical spectra were projected onto the calculated kinematical curve (Fig. 4, left panel).

The active part of MWPC contains three measuring planes: one with vertical wires, one with horizontal wires and one with wires inclined by $45^{\circ}$. Position sensitive efficiency of each plane was obtained using the information from the remaining two others. In order to avoid ambiguities, only events with a single detected particle (confirmed by corresponding $E$ and $\Delta E$ scintillators) were used. Position sensitive efficiency of $\Delta E$ detector was calculated in the similar way but using particles seen in all three planes of the MWPC and $E$ detectors. Since $\Delta E$ detector is used for particle identification, in order to obtain its efficiency for a specific particle (protons or deuterons) one has to use only events identified as elastic scattering, ignoring information from $\Delta E$ detector for one particle. Fig. 3 presents the combined efficiency map of MWPC and $\Delta E$ for protons (left panel) and a sample of unnormalized differential cross section accounting for particle dependent efficiency correction (Fig. 4 right panel). The data points were scaled to the predictions obtained with the CD-Bonn $N N$-potential combined with TM99 $3 N F$. 

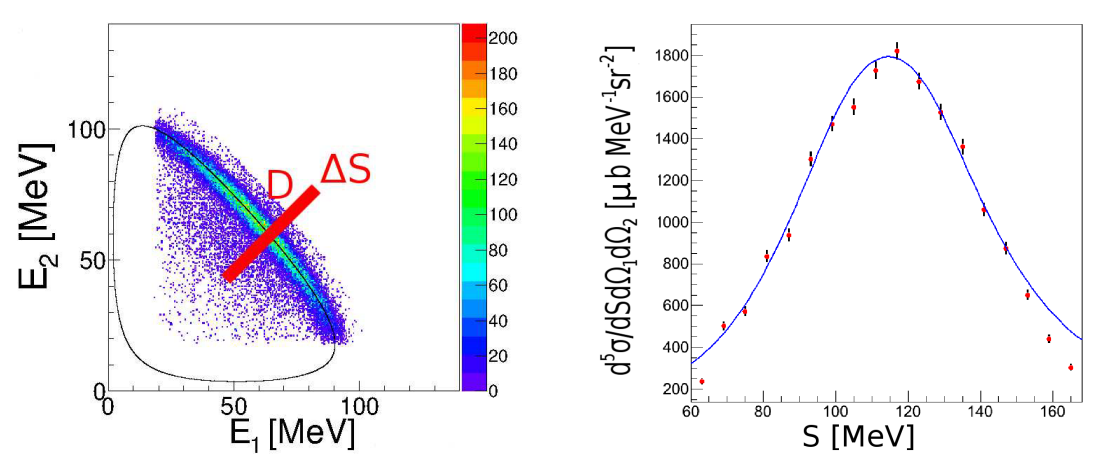

Figure 4. Left panel: Energy versus energy for two protons originating from the breakup reaction with $\theta_{1}=25^{\circ}$, $\theta_{2}=15^{\circ}, \Delta \phi=80^{\circ}$. Right panel: Unnormalised cross section data (red points )compared to the CD-Bonn + TM99 absolute predictions (solid line), for the given configuration.

\section{Acknowledgements}

We acknowledge support by the Foundation for Polish Science - MPD program, co-financed by the European Union within the European Regional Development Fund, Małopolskie Centrum Przedsiębiorczości Project "Doctus - Małopolski fundusz stypendialny dla doktorantów", the Polish 2013-2015 science founds as research Project No. 2012/05/E/ST2/02313 and the Polish National Science Center under Grant No.DEC2013/10/M/ST2/00420. This work was supported by the European Commission within the Seventh Framework Programme through IA-ENSAR (contract no. RII3-CT-2010-262010).

\section{References}

[1] W. Glöckle et al., Phys. Rep. 274107 (1996),

[2] A. Deltuva et al., Phys. Rev. C 68024005 (2003),

[3] E. Epelbaum, Prog. Part. Nucl. Phys. 57654 (2006),

[4] A. Deltuva et al., Phys. Rev. C 73057001 (2006),

[5] H. Witała et al., Phys. Rev. C 83, 044001 (2011),

[6] St. Kistryn et al., Phys. Lett. B 64123 (2006),

[7] E. Stephan at al., Int. J. Mod. Phys. A 24515 (2009),

[8] N. Kalantar-Nayestanaki et al., Rep. Progr. Phys. 75016301 (2012),

[9] W. Parol et al., Acta Phys. Pol. B 451001 (2014),

[10] J. Płoskonka et al., Nucl. Instr. Meth. 12657 (1975). 